\title{
Diagnosis of enteric fever in the emergency department: a retrospective study from Pakistan
}

\author{
Uzma Rahim Khan • Junaid A. Razzak • \\ Ali Faisal Saleem • Adnan Ahmed • Sabeena Jalal
}

Received: 29 May 2009 /Accepted: 23 November 2009/Published online: 23 March 2010

(C) Springer-Verlag London Ltd 2010

\begin{abstract}
Background Enteric fever is one of the top differential diagnoses of fever in many parts of the world. Generally, the diagnosis is suspected and treatment is initiated based on clinical and basic laboratory parameters.

Aims The present study identifies the clinical and laboratory parameters predicting enteric fever in patients visiting the emergency department of a tertiary care hospital in Pakistan.

Methods This is a retrospective chart review of all adult patients with clinically suspected enteric fever admitted to the hospital through the emergency department during a 5year period (2000-2005).

Results A total of 421 emergency department patients were admitted to the hospital with suspected enteric fever. There were 53 cases of blood culture-positive enteric fever and 296 disease-negative cases on culture. The mean age in the blood culture-positive group was 27 years (SD: 10) and in
\end{abstract}

The views expressed in this paper are those of the author(s) and not those of the editors, editorial board or publisher.

U. Rahim Khan · J. A. Razzak ( $₫)$

Department of Emergency Medicine, The Aga Khan University,

Stadium Road, P.O. Box 3500, Karachi 74800, Pakistan

e-mail: junaid.razzak@aku.edu

\section{A. F. Saleem}

Department of Paediatrics, Aga Khan University,

Karachi, Pakistan

\author{
A. Ahmed \\ Pitt County Memorial Hospital, \\ Greenville, NC, USA \\ S. Jalal \\ Harvard School of Public Health, \\ Boston, MA, USA
}

the group with negative blood culture for enteric fever, 35 years (SD: 15) with a male to female ratio of 1:0.6 in both groups. Less than half (48\%) of all patients admitted with suspected enteric fever had the discharge diagnosis of enteric fever, of which only $13 \%$ of the patients had blood culture/serologically confirmed enteric fever. None of the common clinical and laboratory parameters differed between enteric fever-positive patients and those without it. Conclusion Commonly cited clinical and laboratory parameters were not able to predict enteric fever.

Keywords Enteric fever Pakistan - Likelihood ratio · Emergency department

\section{Introduction}

Enteric fever (EF) encompasses both typhoid and paratyphoid fevers. Typhoid fever is caused by Salmonella typhi, whereas paratyphoid fever is caused by $S$. paratyphi $A, B$, and $C$ [1]. Occurring largely in low income countries [2, 3], EF causes 16 million illnesses and 600,000 annual deaths worldwide [4]. It is one of the top differential diagnoses in patients with fever without an obvious source in many parts of the world. In high income countries, EF is suspected in patients with fever and recent history of travel to endemic areas [5].

The diagnosis of EF in the emergency department (ED) is based on clinical signs and symptoms, with basic or no laboratory testing. Neither the sensitivity of initial diagnosis by the physician is known nor is the diagnostic accuracy of clinical features used for such a diagnosis. This study explores the sensitivity, specificity, positive predictive value, and negative predictive value of common clinical and laboratory parameters used for diagnosing EF in the ED. 
Fig. 1 Enteric patients enrolled in the study from the ED of the hospital (July 2000June 2005)

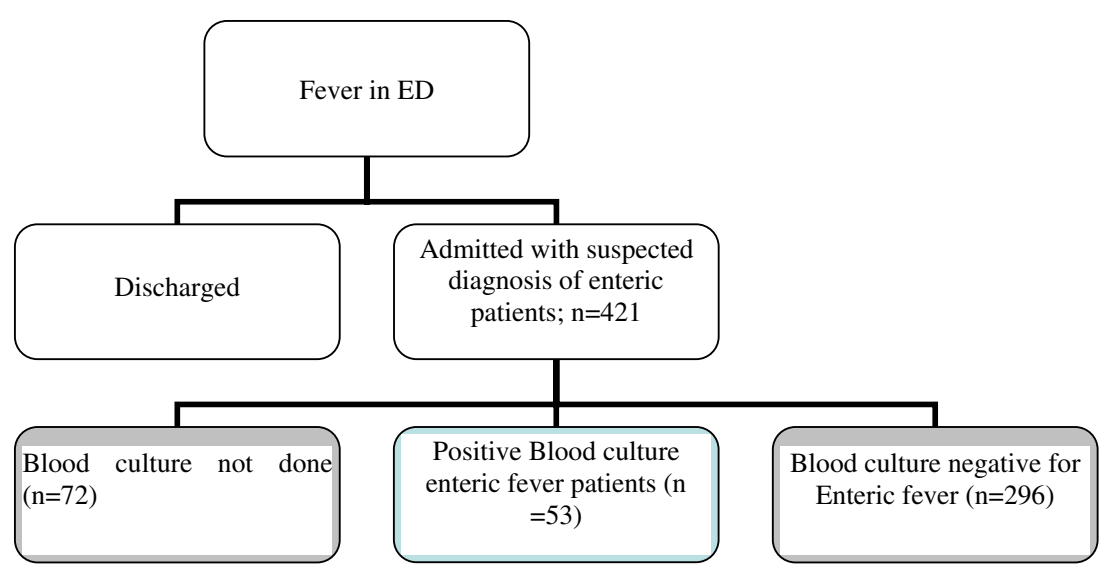

\section{Methods}

\section{Study design}

A retrospective chart review study was conducted.

Study setting and sample

The Aga Khan University Hospital (AKUH) is a private, 540-bed tertiary care teaching hospital, located in Karachi, Pakistan. It is one of the many tertiary care hospitals serving a city of 17 million people. The Emergency Department at AKUH treats about 45,000 patients per year with an admission rate of $35 \%$. Care is primarily provided by residents and medical officers under the supervision of senior faculty members. All patients with an admission diagnosis of suspected EF during the 5-year period (July 2000-June 2005) were included in the study (Fig. 1). The study was approved by the Ethics Review Committee of the Aga Khan University.

\section{Study protocol}

The hospital's health information system was used to identify patients admitted to the hospital through the ED with a suspected diagnosis of EF. Medical records were reviewed by trained research assistants. The completed questionnaires were rechecked by the principal investigator for missing information. Information was extracted on: age, gender, presenting signs and symptoms, comorbidities (for example, hypertension and diabetes), and laboratory parameters (for example, hemoglobin, white cell counts, sodium, potassium, and bicarbonate). Diagnosis of typhoid fever was confirmed by blood culture. Blood cultures are the standard diagnostic method. The sensitivity and specificity for identifying blood culturepositive cases of typhoid fever are 89 and 53\%, respectively $[6,7]$.
Data analysis

The data were analyzed using the Statistical Package for the Social Sciences (SPSS) version 14.0. Descriptive statistics were computed for categorical variables by computing their frequencies. The distribution of quantitative variables was assessed by computing their means and standard deviations. Some of the variables were not included in the analysis due to sparse cell count (for example, coated tongue, confusion, and vertigo). Sensitivity, specificity, positive predictive value (PPV), negative predictive value (NPV), and likelihood ratios (LRs) with 95\% confidence intervals (CIs) were calculated using standard formulas taking blood culturepositive typhoid cases as confirmatory tests (Table 1).

\section{Results}

A total of 421 ED patients were admitted to the hospital with suspected EF. There were 53 cases of blood culturepositive EF and 296 EF-negative cultures. In 72 cases,

Table 1 Measures of diagnostic test accuracy

Measures

Blood culture-positive EF patients and clinical symptom/sign positive (a)

Blood culture-negative and clinical symptom/sign positive (b)

Blood culture-positive EF patients/clinical symptoms and signs negative (c)

Blood culture-negative and clinical symptoms/signs negative (d)

Sensitivity $=(\mathrm{a}) /(\mathrm{a})+(\mathrm{c})$

Specificity $=(\mathrm{d}) /(\mathrm{d})+(\mathrm{b})$

Positive predictive value $=(a) /(a)+(b)$

Negative predictive value $=(\mathrm{d}) /(\mathrm{d})+(\mathrm{c})$

$+\mathrm{LR}=[($ Sensitivity $) /(1-$ Specificity $)]$

$-\mathrm{LR}=[(1-$ Sensitivity $) /$ Specificity $]$ 
Table 2 Comparison of laboratory indices among patients in the different groups (July 2000-June 2005)

\begin{tabular}{|c|c|c|c|c|c|}
\hline \multirow[t]{2}{*}{ Laboratory indices } & \multicolumn{2}{|c|}{ Blood culture positive for EF, $n=53$} & \multicolumn{2}{|c|}{ Blood culture negative for $\mathrm{EF}, n=296$} & \multirow[b]{2}{*}{$p$} \\
\hline & Number & Mean & Number & Mean & \\
\hline Hemoglobin (g/dl) & 52 & 13.1 & 293 & 13 & $=0.8$ \\
\hline WBC $\left(\times 10^{9} / 1\right)$ & 53 & 5.3 & 293 & 6.7 & $=0.001$ \\
\hline Platelets $\left(\times 10^{9} / 1\right)$ & 52 & 166 & 285 & 178 & $=0.3$ \\
\hline Sodium (mmol/1) & 52 & 134 & 277 & 134 & $=0.9$ \\
\hline Potassium (mmol/l) & 52 & 3.7 & 277 & 3.8 & $=0.4$ \\
\hline Chloride (mmol/l) & 34 & 103 & 191 & 104 & $=0.06$ \\
\hline Bicarbonate $(\mathrm{mmol} / \mathrm{l})$ & 37 & 22 & 197 & 21.6 & $=0.5$ \\
\hline Creatinine (mg/dl) & 47 & 1.09 & 255 & 1.1 & $=0.8$ \\
\hline Urea (mg/dl) & 39 & 15.2 & 229 & 12 & $=0.3$ \\
\hline
\end{tabular}

blood cultures were not performed. The mean age in the blood culture-positive group $(n=53)$ was 27 years (SD: $10)$ and in the blood culture-negative group $(n=296)$, 35 years (SD: 15$)$ with a male to female ratio of 1:0.6 in both groups. The most common presenting symptoms of EF cases and disease-negative cases, respectively, were: vomiting $(77 \%, 54 \% ; p=0.002)$, abdominal pain $(19 \%$, $30 \% ; p=0.1)$, chills $(26 \%, 30 \% ; p=0.7)$, diarrhea $(17 \%$, $19 \% ; p=0.7)$, and cough/flu $(13 \%, 21 \% ; p=0.2)$. The abnormal vital signs reported were: tachycardia $(57 \%$, $45 \% ; p=0.1)$, tachypnea $(64 \%, 55 \% ; p=0.2)$, and systolic blood pressure $>120(24 \%, 22 \% ; p=0.6)$. The mean temperature at presentation in both groups was $38^{\circ} \mathrm{C}$ (SD: 1) and mean duration of fever was 7 days (SD: 6) in the first group and 14 days (SD: 33 ) in the second group.
On physical examination, dehydration $(45 \%, 33 \% ; p=$ $0.09)$, anemia $(13 \%, 23 \% ; p=0.2)$, and palpable lymph nodes $(6 \%, 5 \% ; p=0.8)$ were the most common findings in both groups. Hypertension $(2 \%, 10 \% ; p=0.06)$ and diabetes $(4 \%, 8 \% ; p=0.3)$ were the most common comorbidities in the EF-positive and EF-negative groups. There were 2 EF cases with abnormal liver function tests (LFTs) compared to 14 in the disease-negative group ( $p=$ 0.7). Disseminated intravascular coagulation (DIC) was observed in one EF case compared to none in the diseasenegative group. Less than half of the patients had taken antibiotics (penicillin, quinolones, cephalosporin, amino glycosides, sulfonamide, Flagyl, and chloramphenicol) prior to their visit to the ED; the intake percentage in both groups was 41 and $43 \% ; p=0.7$. The laboratory

Table 3 Likelihood ratios of clinical and laboratory parameters of blood culture-positive EF $(n=53)$ versus blood culture negative for EF $(n=296)$ (July 2000-June 2005)

\begin{tabular}{lllllll}
\hline Parameters & Sensitivity & Specificity & PPV & NPV & + LR (95\% CI) & - LR (95\% CI) \\
\hline Abdominal pain & 0.89 & 0.17 & 0.29 & 0.81 & $1.08(0.99-1.18)$ & $0.59(0.31-1.13)$ \\
Epigastric pain & 0.03 & 0.92 & 0.71 & 0.14 & $0.44(0.14-1.37)$ & $1.04(0.96-1.13)$ \\
Anorexia & 0.82 & 0.15 & 0.06 & 0.92 & $0.97(0.80-1.17)$ & $1.15(0.45-2.92)$ \\
Vomiting & 0.54 & 0.22 & 0.79 & 0.08 & $0.68(10.58-0.83)$ & $2.02(1.21-3.38)$ \\
Diarrhea & 0.86 & 0.15 & 1.92 & 0.83 & $1.02(0.91-1.13)$ & $0.87(0.45-1.70)$ \\
Chills & 0.86 & 0.15 & 0.20 & 0.73 & $1.01(0.92-1.12)$ & $0.89(0.50-1.57)$ \\
Cough & 0.20 & 0.86 & 0.89 & 0.16 & $1.56(0.75-3.22)$ & $0.91(0.81-1.03)$ \\
Headache & 0.89 & 0.15 & 0.11 & 0.92 & $1.06(0.94-1.19)$ & $0.66(0 . .25-1.74)$ \\
Past history of EF & 0.77 & 0.14 & 0.04 & 0.92 & $0.91(0.71-1.17)$ & $1.50(0.60-3.69)$ \\
Dehydration & 0.33 & 0.54 & 0.80 & 0.12 & $0.73(0.52-1.02)$ & $1.22(0.94-1.58)$ \\
Anemia & 0.21 & 0.86 & 0.9 & 0.16 & $1.61(0.78-3.32)$ & $0.90(0.80-1.02)$ \\
Palpable lymph nodes & 0.05 & 0.94 & 0.83 & 0.15 & $0.89(0.26-2.98)$ & $1.00(0.93-1.08)$ \\
Leukopenia & 0.31 & 0.77 & 0.88 & 0.17 & $1.36(0.80-2.30)$ & $0.89(0.76-1.05)$ \\
Neutropenia & 0.31 & 0.77 & 0.88 & 0.16 & $1.37(0.81-2.32)$ & $0.89(0.75-1.05)$ \\
Thrombocytopenia & 0.42 & 0.59 & 0.85 & 0.15 & $1.05(0.74-1.51)$ & $0.95(0.75-1.22)$ \\
\hline
\end{tabular}


results in the ED showed an average WBC count of 5.3 (SD: 2) and 6.6 (SD: 4) $\times 10^{9} / 1$, hemoglobin of 13 (SD: 1 and 2) $\mathrm{g} / \mathrm{dl}$, and a platelet count of 166 (SD: 63) and 178 $(\mathrm{SD}: 107) \times 10^{9} / 1$ (Table 2).

Of all 421 patients suspected of having EF, a little less than half $(48 \%)$ were discharged with the diagnosis of EF. The diagnoses of the remaining $52 \%$ patients were viral fever $(21 \%)$, malaria $(6 \%)$, invasive gastroenteritis $(5 \%)$, urinary tract infection (4\%), and upper respiratory tract infection $(3 \%)$.

Individual clinical and laboratory findings in the ED did poorly in differentiating patients of the two groups. Diarrhea LR $(+1.02$, 95\% CI: 0.91-1.13), dehydration LR $(+0.73$, 95\% CI: $0.52-1.02)$, leukopenia LR $(+1.36,95 \%$ CI: $0.80-2.30)$, and abdominal pain LR $(+1.08,95 \%$ CI: $0.99-1.18)$ etc. were not able to differentiate between these two groups (Table 3).

\section{Discussion}

Our study shows that in an endemic country like Pakistan, about less than half of patients admitted with the diagnosis of suspected EF actually have EF. In nonendemic parts of the world, clinical diagnostic sensitivity is likely to be much lower. In this study, one of the largest ED-based studies, no single clinical or laboratory indicator had a positive LR high enough to help clinical decision-making.

A number of small, non-ED-based studies have looked at the clinical diagnosis of EF. In Indonesia, in a prospective outpatient clinic-based study of 82 pediatric and adult typhoid/paratyphoid patients, Vollaard et al. found a low sensitivity of presenting symptoms. The study failed to find a clinical prediction rule [8]. Similarly, in Nepal, a prospective observational study conducted at a teaching hospital emergency and outpatient department showed that the majority of the symptoms and signs of typhoid in 53 adult cases were without a very high diagnostic accuracy [9]. Many other studies failed to show much difference in the clinical profiles of patients [10-17], though none of these studies evaluated the accuracy of emergency physicians in diagnosing EF.

There were at least two studies where some clinical and laboratory features were found to be highly predictive. In Bangladesh, Haq et al. prospectively studied 106 adult patients with microbiologically confirmed EF comparing them to 170 adult patients with other established febrile illnesses. The study found that history of a stepladder pattern of rise in temperature, loose motions, relative bradycardia, and coated tongue proved to be powerful markers of EF with high specificity $(100,94.7,94.7$, and $94.1 \%$, respectively) [18]. In a study of 130 adult cases,
Hosoglu et al. created a prediction rule using seven predictors. These predictors were age $<30$ years, abdominal distention, confusion, leukopenia, relative bradycardia, positive Widal test, and a typhoid tongue [19]. This prediction rule was validated in the same region where it was developed.

\section{Limitations}

There are several limitations of our study. First, this was a single-center study and may not represent the findings at other centers in Karachi or Pakistan. Second, being an EDbased study, our findings are likely to be applicable to more severe cases as only those who required admission to the hospital were included in this study. Third, due to the retrospective nature of the study, we were limited not only by the completeness of documentation by the treating physician but were also not able to assess all the variables (for example, relative bradycardia). Fourth, there may have been other patients with $\mathrm{EF}$ admitted through the ED, but because the clinician did not consider this diagnosis at the time of admission they were not included in this analysis. Fifth, a large number of blood culture negatives could be due to the fact that sensitivity of blood cultures decreases after the first week of illness and also due to prior use of an antibiotic.

\section{Conclusion}

In endemic settings, accurate diagnosis of EF via clinical and laboratory findings is difficult in an ED of a tertiary care teaching hospital.

Conflicts of interest None.

\section{References}

1. WHO (2008) Enteric fever. In: Guidelines on standard operating procedures for microbiology. WHO Regional Office for SouthEast Asia, Geneva, Switzerland

2. González A, Malo Q, Román Muñoz J, Molina J, Pérez MT, Domínguez J, Bisquert J (1994) Microbiologic diagnosis of typhoid fever: report on an outbreak (in Spanish). Enferm Infecc Microbiol Clin 12:372-377

3. Crum NF (2003) Current trends in typhoid fever. Curr Gastroenterol Rep 5:279-286

4. Crump JA, Luby SP, Mintz ED (2004) The global burden of typhoid fever. Bull World Health Organ 82:346-353

5. CDC (2007) Prevention of specific infectious diseases: typhoid fever, Chap. 4. In: Arguin PM, Kozarsky PE, Reed C (eds) CDC health information for international travel 2008. Elsevier, Atlanta

6. Bhan MK, Bahl R, Bhatnagar S (2005) Typhoid and paratyphoid fever. Lancet 366:749-762 
7. Parry CM, Hien TT, Dougan G, White NJ, Farrar JJ (2002) Typhoid fever. N Engl J Med 347:1770-1782

8. Vollaard AM, Ali S, Widjaja S, Asten HA, Visser LG, Surjadi C, van Dissel JT (2005) Identification of typhoid fever and paratyphoid fever cases at presentation in outpatient clinics in Jakarta, Indonesia. Trans R Soc Trop Med Hyg 99:440-450

9. Neopane APM, Pradhan B, Dhakal R, Karki DB (2006) Enteric fever: diagnostic value of clinical features. Kathmandu Univ Med J 4:307-315

10. Nazar HS, Rabbani A, Riaz A, Anwar J (2005) Presentation of typhoid fever patients in Hazara Division and response to different treatment regimens. J Ayub Med Coll Abbottabad 17:67-69

11. Gendron Y, Thevenieau D, Touze JE, Mailloux C, Barabe P (1981) Typhoid fever in adult patients in Ouagadougou (analytic study of 124 cases) (in French). Med Trop (Mars) 41:625-631

12. Ross IN, Abraham T (1987) Predicting enteric fever without bacteriological culture results. Trans R Soc Trop Med Hyg 81:374-377

13. Khan M, Coovadia YM, Connoly C, Sturm AW (1998) The early diagnosis of typhoid fever prior to the Widal test and bacteriological culture results. Acta Trop 69:165-173

14. Mathura KC, Gurubacharya DL, Shrestha A, Pant S, Basnet P, Karki DB (2003) Clinical profile of typhoid patients. Kathmandu Univ Med J 1:135-137

15. Tohme A, Zein E, Nasnas R (2004) Typhoid fever. Clinical and therapeutic study in 70 patients (in French). J Med Liban 52:71-77
16. Su CP, Chen YC, Chang SC (2004) Changing characteristics of typhoid fever in Taiwan. J Microbiol Immunol Infect 37:109-114

17. Lefebvre N, Gning SB, Nabeth P, Ka S, Ba-Fall K, Rique M, Sane M, Chevalier B, Mbaye PS, Debonne JM (2005) Clinical and laboratory features of typhoid fever in Senegal. A 70-case study (in French). Med Trop (Mars) 65:543-548

18. Haq SA, Alam MN, Hossain SM, Ahmed T, Tahir M (1997) Value of clinical features in the diagnosis of enteric fever. Bangladesh Med Res Counc Bull 23:42-46

19. Hosoglu S, Celen MK, Geyik MF, Akalin S, Ayaz C, Acemoglu H, Loeb M (2006) Risk factors for typhoid fever among adult patients in Diyarbakir, Turkey. Epidemiol Infect 134:612-616

Uzma Rahim Khan is a Senior Instructor for research in the Department of Emergency Medicine. She has done an MBBS from Sind Medical College of Karachi and a Masters in Epidemiology and Biostatistics from the Aga Khan University. Her area of interest is epidemiological studies related to trauma, injury, school injuries, reproductive health, and infectious diseases. She is responsible for providing epidemiological and statistical consultation for various research projects of the department. This also includes proposal and manuscript writing.Currently, she has three publications to her credit and about five projects in progress. 